\title{
Origin of Chemiluminescence Accompanying the Reaction of the 9-Cyano-10-methylacridinium Cation with Hydrogen Peroxide
}

\author{
A. Wróblewska, ${ }^{1}$ O.M. Huta, ${ }^{2}$ S.V. Midyanyj, ${ }^{2}$ I.O. Patsay, ${ }^{2}$ J. Rak, ${ }^{1}$ and J. Błażejowski ${ }^{1, *}$ \\ ${ }^{1}$ Faculty of Chemistry, University of Gdańsk, J. Sobieskiego 18, 80-952 Gdańsk, Poland \\ ${ }^{2}$ Faculty of Chemistry, Ivan Franko L'viv National University, Kyrylo and Mefodiy 6, 79005 \\ L'viv, Ukraine
}

\section{Supporting Information}

In Table S1 (page S2) the energies of stationary points and the zero-point energies (both in Hartrees) for DFT calculations are shown as well as the number of imaginary frequencies.

In Figures S1 (page S3) and S2 (page S4), the PM3 and DFT optimized geometries of molecules and the entities relevant to the transition states, respectively, are presented.

Additionally, in separated file named "atomic coordinates.rtf", the geometries of all stationary points are given in Cartesian coordinates (in $\AA$ ). 
Table S1. Energies and zero-point energies of the stationary points and the number of imaginative frequencies

\begin{tabular}{|c|c|c|c|c|}
\hline Entity & Method $^{a}$ & $\mathrm{E}^{b}$ & $\mathrm{ZPE}^{c}$ & $\mathrm{NIMAG}^{d}$ \\
\hline \multicolumn{5}{|c|}{$\mathrm{S}_{0}$ state } \\
\hline \multirow[t]{2}{*}{1} & DFT & -687.511575777 & 0.222738 & 0 \\
\hline & PM3 & & & 0 \\
\hline \multirow[t]{2}{*}{3} & DFT & -838.670564739 & 0.239441 & 0 \\
\hline & PM3 & & & 0 \\
\hline 4 & PM3 & & & 0 \\
\hline \multirow[t]{2}{*}{5} & DFT & -838.634468907 & 0.240943 & 0 \\
\hline & PM3 & & & 0 \\
\hline \multirow[t]{2}{*}{7} & DFT & -763.542282469 & 0.236278 & 0 \\
\hline & PM3 & & & 0 \\
\hline 8 & PM3 & & & 0 \\
\hline \multirow[t]{2}{*}{9} & DFT & -763.531603765 & 0.236923 & 0 \\
\hline & PM3 & & & 0 \\
\hline \multirow[t]{2}{*}{10} & DFT & -838.675504345 & 0.241146 & 0 \\
\hline & PM3 & & & 0 \\
\hline \multirow[t]{2}{*}{11} & DFT & -838.081597967 & 0.225647 & 0 \\
\hline & PM3 & & & 0 \\
\hline 13 & PM3 & & & 0 \\
\hline \multirow[t]{2}{*}{16} & DFT & -670.130875307 & 0.216123 & 0 \\
\hline & PM3 & & & 0 \\
\hline \multirow[t]{2}{*}{17} & DFT & -914.485290900 & 0.252094 & 0 \\
\hline & PM3 & & & 0 \\
\hline \multirow[t]{2}{*}{20} & DFT & -762.965348130 & 0.221583 & 0 \\
\hline & PM3 & & & 0 \\
\hline \multirow[t]{2}{*}{22} & DFT & -839.395291877 & 0.249435 & 0 \\
\hline & PM3 & & & 0 \\
\hline $3 \rightarrow 10$ & PM3 & & & 1 \\
\hline $11 \rightarrow 13$ & PM3 & & & 1 \\
\hline $13 \rightarrow 15+16$ & PM3 & & & 1 \\
\hline $11 \rightarrow 15+16$ & DFT & -838.074756417 & 0.225023 & 1 \\
\hline \multirow[t]{2}{*}{$17 \rightarrow 12+15+16$} & DFT & -914.475659577 & 0.250616 & 1 \\
\hline & PM3 & & & 1 \\
\hline \multirow[t]{2}{*}{$10 \rightarrow 16+18$} & DFT & -838.625972432 & 0.236785 & 1 \\
\hline & PM3 & & & 1 \\
\hline \multirow[t]{2}{*}{$7 \rightarrow 16+19$} & DFT & -763.485505153 & 0.233075 & 1 \\
\hline & PM3 & & & 1 \\
\hline \multirow[t]{2}{*}{$20 \rightarrow 16+21$} & DFT & -762.960588244 & 0.220658 & 1 \\
\hline & PM3 & & & 1 \\
\hline \multirow[t]{2}{*}{$3 \rightarrow 16+18$} & DFT & -838.585936371 & 0.236373 & 1 \\
\hline & PM3 & & & 1 \\
\hline \multirow[t]{2}{*}{$22 \rightarrow 12+16+21$} & DFT & -839.373042759 & 0.247705 & 1 \\
\hline & PM3 & & & 1 \\
\hline \multicolumn{5}{|c|}{$\mathrm{S}_{1}$ state } \\
\hline 14 & $\mathrm{PM} 3 / \mathrm{CI}$ & & & 0 \\
\hline
\end{tabular}

\footnotetext{
${ }^{a} \mathrm{DFT}=\mathrm{B} 3 \mathrm{LYP} / 6-31 \mathrm{G} * * / / \mathrm{B} 3 \mathrm{LYP} / 6-31 \mathrm{G}^{* *}$

${ }^{b}$ Energy (in Hartrees)

${ }^{c}$ Zero-point energy (in Hartrees)

${ }^{d}$ Number of imaginative frequencies
} 
Figure S1. PM3 and DFT optimized geometries of molecules (Schemes 1 and 2) originating from acridine that occur along the pathway of the reaction of $\mathrm{CMA}^{+}$with $\mathrm{OOH}^{-}$and $\mathrm{OH}^{-}$.

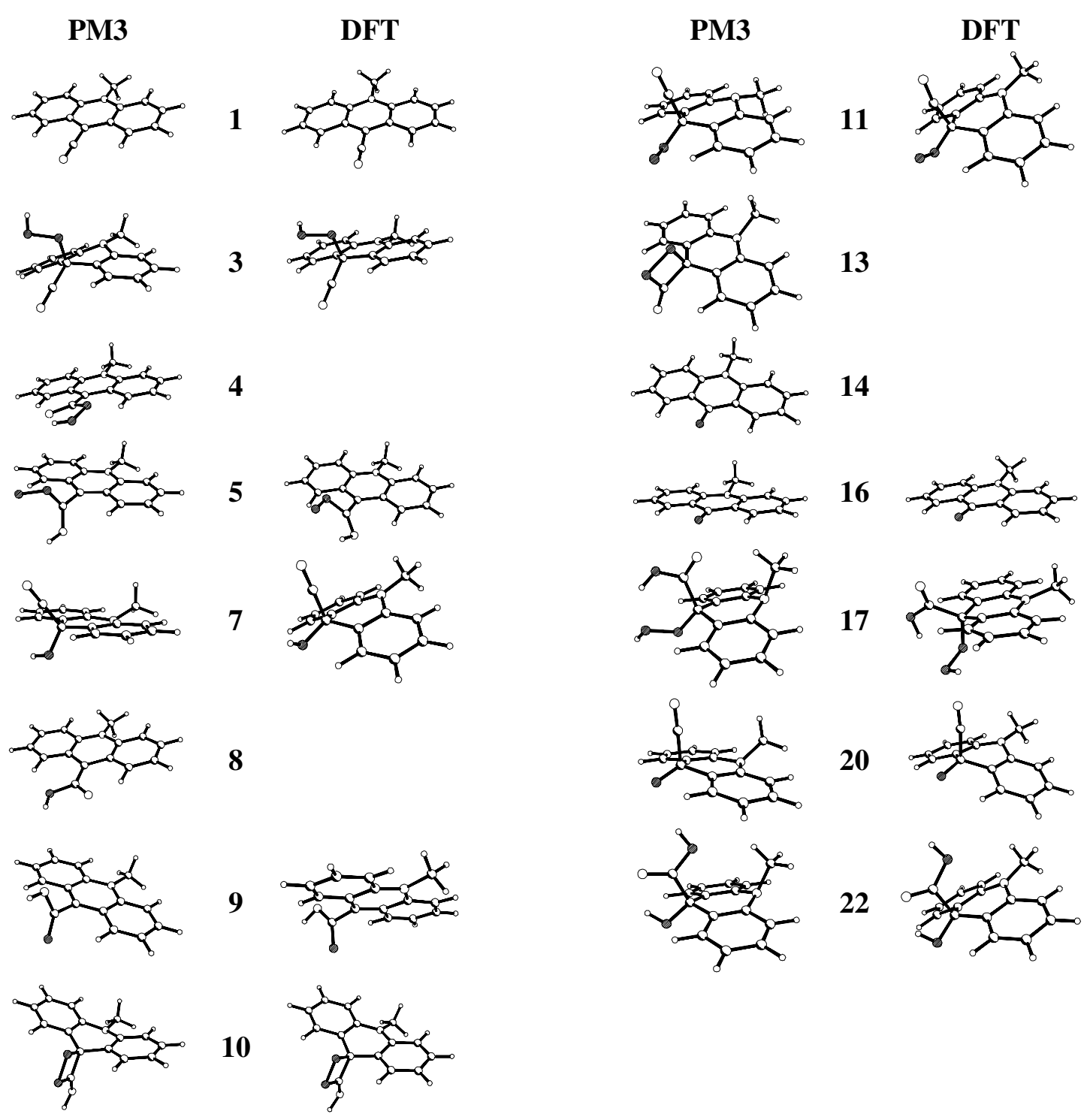


Figure S2. PM3 and DFT optimized geometries of the entities relevant to the transition states (Schemes 1 and 2) occurring along the pathway of the reaction of $\mathrm{CMA}^{+}$with $\mathrm{OOH}^{-}$and $\mathrm{OH}^{-}$. The dotted lines represent the bonds that are formed or broken during the course of the reaction.

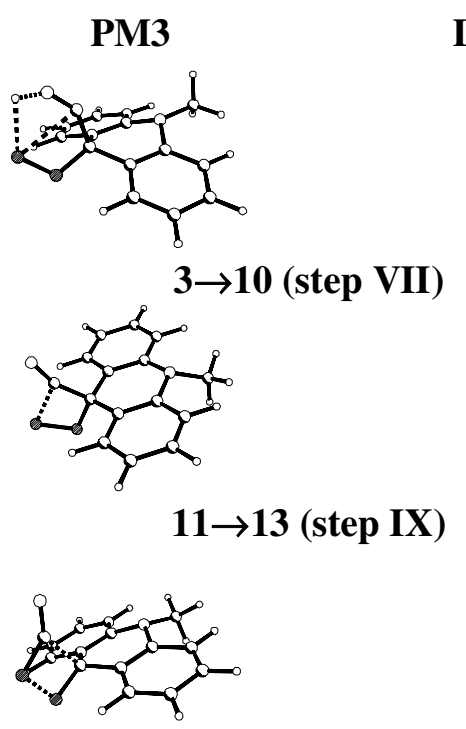

DFT

$$
13 \rightarrow 15+16(\text { step } X)
$$

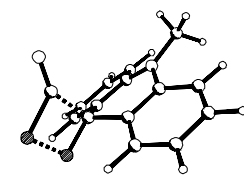

$11 \rightarrow 15+16$ (step XII)
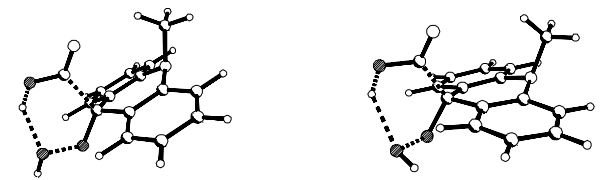

$17 \rightarrow 12+15+16$ (step XIV)
PM3

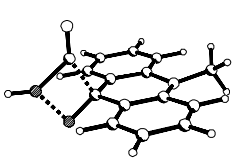

$3 \rightarrow 16+18$ (step XV)
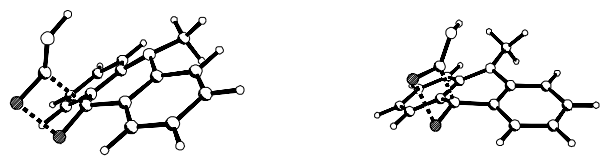

$10 \rightarrow 16+18$ (step XVIII)
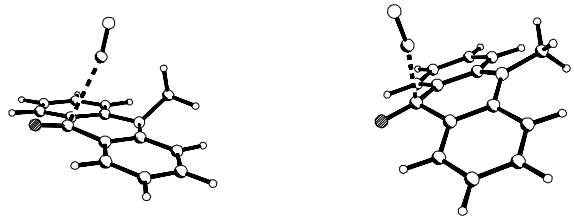

$20 \rightarrow 16+21($ step XX)
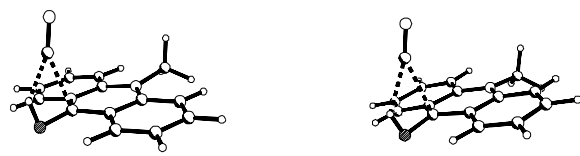

$7 \rightarrow 16+19$ (step XXI)
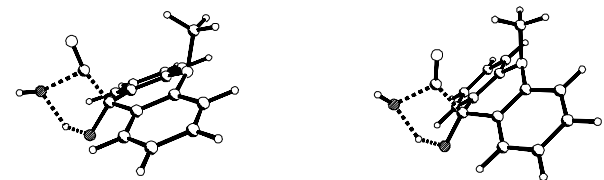

$22 \rightarrow 12+16+21$ (step XXIII) 